\title{
Atypical Language Development in \\ Romance Languages
}

Edited by

Eua Aguilar-Mediavilla, Lucía Buil-Legaz,

Raúl López-Penadés, Victor A. Sanchez-Azanza and Daniel Adrover-Roig

\section{John Benjamins Publishing Company}


Atypical Language Development in Romance Languages 


\section{Atypical Language Development in Romance Languages}

Edited by

Eva Aguilar-Mediavilla

Lucía Buil-Legaz

Raúl López-Penadés

Victor A. Sanchez-Azanza

Daniel Adrover-Roig

University of Balearic Islands

John Benjamins Publishing Company

Amsterdam / Philadelphia 
The paper used in this publication meets the minimum requirements of the American National Standard for Information Sciences - Permanence of Paper for Printed Library Materials, ANSI z39.48-1984.

DOI $10.1075 /$ Z.223

Cataloging-in-Publication Data available from Library of Congress: LCCN 2019005526 (PRINT) / 2019015426 (E-BOOK)

ISBN 9789027203212 (HB)

ISBN 9789027262493 (Е-BOOK)

(C) 2019 - John Benjamins B.V.

This e-book is licensed under a Creative Commons CC BY-NC-ND license. To view a copy of this license, visit https://creativecommons.org/licenses/by-nc-nd/4.o/. For any use beyond this license, please contact the publisher.

John Benjamins Publishing Company · https://benjamins.com 


\section{Table of contents}

Introduction to atypical language development in romance languages

Eva Aguilar-Mediavilla, Lucía Buil-Legaz, Raúl López-Penadés,

Víctor A. Sánchez-Azanza and Daniel Adrover-Roig

\section{Part I. Preterm children}

Neuroconstructivism to understand the effect of very preterm birth on language and literacy

Annalisa Guarini, Mariagrazia Zuccarini and Alessandra Sansavini

Prematurity, executive functions and language: A study with low risk preterm children

Miguel Pérez-Pereira, Manuel Peralbo and Alberto Veleiro

Risk for language delay in healthy preterm and full-term children:

A longitudinal study from 22 to 60 months

Mariela Resches, Miguel Pérez-Pereira, Raquel Cruz Guerrero and

Montse Fernández Prieto

Word segmentation and mapping in early word learning: Differences between full term and moderately preterm infants

Laura Bosch, Maria Teixidó and Thais Agut

\section{Part II. Specific Language Impairment}

The influence of maternal education on the linguistic abilities of monolingual Spanish-speaking children with and without Specific Language Impairment

Alejandra Auza-Benavides, Christian Peñaloza C. and Chiharu Murata

Idiom understanding competence of Spanish children with Specific

Language Impairment and Pragmatic Language Impairment 
Evaluation of narrative skills in language-impaired children: Advantages of a dynamic approach

Ingrida Balčiūnienè and Aleksandr N. Kornev

Real-time comprehension of sentences in children with SLI: Evidence from eye movements

Llorenç Andreu, Nadia Ahufinger, Laura Ferinu, Fernanda Pacheco, Roser Colomé and Mònica Sanz Torrent

\section{Part III. Deafness}

Emotion recognition skills in children with hearing loss: What is the role of language?

Francesc Sidera, Elisabet Serrat, Anna Amadó and Gary Morgan

Executive functions and eye fixations in children with Cochlear Implant

María Fernanda Lara Díaz, Carolina Rivera and

Silvia Raquel Rodriguez

\section{Part IV. Genetic syndromes with intellectual disabilities}

The relationship between the lexicon and grammar in Spanish-speaking children with Down syndrome

Donna Jackson-Maldonado, Miguel Galeote and

María Fernanda Flores Guerrero

Profiles of grammatical morphology in Spanish-speaking adolescents with Williams Syndrome and Down Syndrome

Eliseo Díez-Itza, Manuela Miranda, Vanesa Pérez and

Verónica Martínez

Evaluative language and component structure of oral narratives in

Williams Syndrome

Marta Shiro, Eliseo Diez-Itza and Maite Fernández-Urquiza 


\title{
Emotion recognition skills in children with hearing loss
}

\section{What is the role of language?}

\author{
Francesc Sidera, ${ }^{1}$ Elisabet Serrat, ${ }^{1}$ Anna Amadó ${ }^{1}$ and \\ Gary Morgan ${ }^{2}$ \\ ${ }^{1}$ Universitat de Girona / ${ }^{2}$ City, University of London
}

\begin{abstract}
Previous studies have found a deficit in emotion recognition skills in children with hearing loss linked to their linguistic development. Our aim is to explore how different linguistic-communicative skills influence the capacity to recognise emotions from faces, at different developmental points, in children with and without hearing loss. We administered language measures and a task of emotion recognition (ER) to 166 children (75 with hearing loss). Results show that ER was linked to various linguistic-communicative skills in children with hearing loss, whereas fewer connections existed in hearing children. As these relations varied with age, we discuss how the importance of the different linguistic and communicative skills for ER varies throughout development and as a function of hearing status.
\end{abstract}

Keywords: emotion recognition, vocabulary, linguistic-communicative skills, hearing loss, children

\section{Introduction}

Several studies have shown that language is important for the development of sociocognitive skills, and particularly, for emotion understanding (e.g. Astington \& Jenkins 1999; Rieffe \& Wiefferink, 2017). It follows that hearing-impaired (from now on, HI) children with linguistic difficulties might have social understanding delays (see, for example, Peterson, Wellman, \& Slaughter, 2012). Such social understanding delays might be explained by difficulties in formal aspects of language (Dyck, Farrugia, Shochet, \& Holmes-Brown, 2004) like vocabulary or grammar development, but also by deficits in early communication abilities that 
emerge out of social interactions and conversations (Meristo, Strid, \& Hjelmquist, 2016; Morgan et al., 2014). In this regard, the objectives of this study are: a) to understand better how different aspects of language are related to the capacity to recognise emotions at different developmental points; and b) to study whether this relationship evolves similarly in children with or without linguistic difficulties.

\section{Deficits in emotion recognition in children with hearing loss}

Controversy exists in relation to whether HI children have a deficit in their capacity to recognise emotions. On the one hand, various studies have found difficulties in recognising facial emotions (for example Dyck et al., 2004; Gray, Hosie, Russell, Banks, \& Ormel, 2001; Wang et al., 2011), even in non-verbal tasks (Wang, Su \& Yan, 2016; Wiefferink et al., 2013). The age of the child at assessment and type of emotion evaluated are very relevant for determining these difficulties. In the study by Sidera et al., (2017) the specific emotions that HI children had difficulty with in comparison to hearing children varied with age. In that study, the young $\mathrm{HI}$ group (3-and 4-year-olds) were significantly delayed in the recognition of fear, the medium aged HI group (5- and 6-year-olds) had difficulty in the recognition of disgust and surprise, and the oldest HI group (7- and 8-year-olds) found recognition of surprise and fear complicated. However, the HI group followed the same developmental order in the recognition of emotions as the hearing comparison group (see also Ziv, Most, \& Cohen, 2013). Wang et al., (2016) report evidence that young HI children (aged 4 years) who have had relatively little experience with aided hearing (because of their age) even struggle to label basic emotions (e.g. happy, sad, fear). According to the developmental model of Widen, and Russel (2013) these basic emotions are the first labels to be acquired in typically developing children. In summary, HI children have been shown to have delays in labeling, emotions.

On the other hand, there are studies that have not found evidence of this delay. This can be attributed to various factors: a) these studies did not include late-acquired labels for emotion faces, such as disgust or surprise (see: Laugen, Jacobsen, Rieffe, \& Wichstrøm, 2017; Mancini et al., 2016); b) these studies involved older study participants (see: Hopyan-Misakyan, Gordon, Dennis, \& Papsin, 2009; Most and Aviner, 2009), who might have caught-up with their peers; or c) these studies compared a group of HI children with a group of hearing children of a younger age (see: Hosie et al., 1998 in their young group; Ziv et al., 2013).

Another important aspect to be considered is the child's level of hearing loss. Some authors have found emotion recognition (ER) difficulties in children with profound hearing loss but not with severe to moderate hearing losses (see for example, Most \& Michaelis, 2012). Methodological aspects also matter, e.g. Jones, 
Gutierrez, and Ludlow (2017) found that HI children (including users of sign language) labelled facial emotional expressions better when they were dynamic (from a video) rather than in static pictures (without movement).

\section{Language and emotion recognition in children with hearing loss}

In wider research, it is thought that the development of language and emotion concepts is interdependent, and language is a powerful tool for developing an understanding of emotion (Mancini et al., 2016). It is possible that different aspects of emotion understanding may be more or less dependent on language (see Dyck et al., 2004). Thus, research on how the linguistic delays of HI children affect their understanding of emotions may help us understand the role that language has in constructing emotional concepts more generally.

However, some researchers have failed to find a relationship between language and ER, even in verbal tasks (Jones et al., 2017), but this study only used one linguistic measure, and did not compare whether HI children were actually linguistically delayed. Other studies have found such a relationship (for example, Dyck et al., 2004). Sidera et al., (2017) found language-related difficulties (in vocabulary and linguistic-communicative skills) in HI children when they were labelling facial emotions depicted in drawings. If language is a likely reason for ER delays in HI children, there may be other important contributors. Sidera et al., (2017) found that even after matching vocabulary, non-verbal reasoning and chronological age, the HI group was still delayed in ER compared to the hearing group. More research is needed to better understand how HI children develop language and ER.

The ability to label and understand emotions at an age appropriate level is important for wider mental health and social development. For example, several studies have linked delays in emotion understanding to risks in the development of cognitive regulation (Carlson \& Wang, 2007; Botting et al., 2016).

In the present study, we extend Sidera et al., (2017) by considering how the relationship between linguistic and ER skills changes across different developmental points in $\mathrm{HI}$ and hearing children. This will allow us to better understand which aspects of language are most connected to HI and ER at different ages. As language and ER skills develop with age, we expect that diverse linguistic components will be linked to ER at different ages. The existent literature does not allow us to make predictions about how these relationships evolve, so in this sense this study is exploratory. 


\section{Methodology}

\section{Participants}

For this study, we used the same sample as in Sidera et al.s (2017) study, which we briefly detail next (see this study for more details). It consisted of 166 children (91 hearing and 75 with hearing loss) aged between 39 and 107 months (Mean $=71.96$ months; $S D=18.39)$. The groups with and without hearing loss were very similar in terms of age (children with hearing loss: Mean $=71.01$ months; $S D=17.87$; hearing children: Mean $=72.84$ months, $S D=18.86$; Mann Whitney's $U=3226.5, p=.546$ ) and cognitive ability (children with hearing loss: Mean $=131.31 ; S D=44.54$; hearing children: Mean $=135.31$, $S D=40.67$; Mann Whitney's $U=3088.5, p=.293$ ). The percentage of boys and girls was also similar in both groups ( $45.3 \%$ of girls in the HI group, and $50.5 \%$ in the hearing group), and the Chi-Square test showed that there were no significant group differences $\left(\chi^{2}=.448 ; p=.503\right)$. Children with reported learning difficulties or other pathologies apart from HI were not included in the sample.

Regarding the characteristics of the HI group, all children had prelocutive (onset before the age of 12 months) bilateral hearing loss and attended mainstream oral schools, which is the most common educational option in Catalonia, the region where data were collected. The mean age of detection of the hearing loss was 19.24 months $(S D=19.43$; range $=0$ to 75$)$, and the mean age of hearing devices fitting was 26.91 months $(S D=18.39$; range $=4$ to 81$)$. From the $75 \mathrm{HI}$ children, 36 had a cochlear implant (with or without additional hearing aids) while 38 had only hearing amplification devices (including here a child with a bone-attached hearing implant); one had never had sensory aids. Speech therapists and teachers reported that none of the children used sign language. As far as the level of hearing loss (in the better ear) is concerned, one child had mild hearing loss (from 21 to $40 \mathrm{~dB}$ of loss), 25 moderate (from 41 to $70 \mathrm{~dB}$ ), 12 severe (from 71 to $90 \mathrm{~dB}$ ) and 37 profound (from $91 \mathrm{~dB}$ ). Level of hearing was reported by the speech therapist of each child through a questionnaire (see materials section).

It is noteworthy that when we compared the sample with and without hearing loss in the linguistic tasks (in expressive vocabulary and linguistic-communicativeskills) we found that the mean scores of HI children in both tasks were located between the percentile 17 and 18 of the scores from our sample of hearing children.

\section{Materials}

Children were evaluated on the following tasks: 
1. Naming vocabulary subtest of the British Ability Scales-2 (BAS-2). In this expressive vocabulary task children have to label pictures (Elliot et al., 1996; Spanish adaptation by Arribas \& Corral, 2011). Depending on the age of the child, children are shown a different set of pictures. We used the aptitude score of the test which transforms the raw score by considering the particular level of difficulty administered to each child.

2. Cognitive ability.

The pattern construction subtest of the BAS-2 (Elliot et al., 1996; Spanish adaptation Arribas \& Corral, 2011) was used to control for the non-verbal reasoning skills of the child. The test is a good proxy of general cognitive ability. In this task, children have to reproduce visual patterns by using squares and/or cubes. Again, as different children may be administered different items according to their age and performance, the aptitude score was used.

3. Facial emotion recognition task.

We administered a facial ER task that required children to identify emotion labels and match them to facial emotional expressions, which may be more dependent on language skills than emotion-matching or emotion discrimination tasks (see: Wang et al., 2016; Rieffe \& Wiefferink, 2017). It consisted of six coloured cartoons of a girl depicting happiness, sadness, fear, anger, surprise and disgust (published in Sidera et al., 2017). All drawings were placed in front of the child (in two lines of three drawings, in random order) and they were asked: "Could you point to the girl looking... and then: happy, sad, scared, angry, surprised or disgusted" (in Catalan language, which corresponds to the labels of "contenta", "trista", "espantada", "enfadada", "sorpresa" and "fàstic"). After children gave an answer, the experimenter only said "Ok" before moving to the next emotion. The order of presentation of the questions was counterbalanced using a Latin-square design. The researcher took notes of children's responses, and awarded 1 point for each correct answer. Their scores in this task varied from 0 to 6 (ER score), corresponding to the number of drawings they correctly pointed to.

4. Questionnaire with sociodemographic and audiological data.

This questionnaire included the following sociodemographic information: date of birth, number of siblings, mother tongue of the mother and father, language used by the mother and father with the child, educational level of the parents, communicative systems (oral language, written language, sign languages, cued speech, lip-reading, or others) used at home and school, preferred communicative system of the child and age of the first word. For the children with hearing loss, the questionnaire also included a part on audiological information, where we asked about the cause of the hearing loss, the level of hearing loss in each ear, the use of hearing devices, the existence 
of relatives with hearing loss and the knowledge of different communication systems. Speech therapists for the HI and teachers for the typically hearing children filled out this information. Take note that not all the information are provided in this paper because it is not needed to our aim.

5. Linguistic Proficiency Profile - 2 (LPP-2).

The same professionals responded to the LPP-2, a tool designed to assess the linguistic-communicative skills of HI children but which is also suitable for hearing children (Bebko \& McKinnon, 1993).

LPP-2 is a measure of general language development (see Bebko, Calderon \& Treder, 2003). The original author gave permission to translate the scale from the Spanish version into the Catalan language. The tool assesses five areas of expressive language and communication skills: Form, Content, Reference, Cohesion, and Use (each subscale has 9,12,11,11 and 13 items, respectively). A person who is familiar with the child's speech has to evaluate their level of mastery for each item, which can be described as: a) not acquired (0 points); b) emerging (1 point); c) or acquired/past (2 points). In the original scale the total score of the scale is 112 . However, since 87 from the 166 participants had, at least, one item not answered (the LPP-2 contemplates that respondents may be insecure about the level of the child), we decided to score the scale with percentages (from these 87 children, the mean number of unanswered items was 2.12, apart from a child whose LPP-2 could not be obtained). Hence, we calculated the percentage of the Total LPP-2 score, as the number of points obtained by the child divided by the maximum number of points they could obtain without considering the unanswered items. So, for example, if a child had one blank item, his percentage of points would be calculated by dividing his total score per 110, instead of per 112 (as each item has a maximum score of 2). The same procedure was followed in each of the 5 LPP-2 subscales. This also permitted us to compare the percentage of points from one subscale to another, as not all subscales have the same number of items.

\section{Procedure}

Parental informed consent was obtained for each child before conducting the study, and permission by the school centers and Catalan Department of Education were also obtained. Children were individually interviewed at their own schools, in a quiet room. Tasks were administered by a researcher in one session lasting from 35 to 55 minutes. Children with hearing loss were administered the tasks with the presence of their speech therapist.

For purposes of data analysis the sample was split into three age groups (young group: 3- and 4-year-olds, medium group: 5- and 6- year olds and old group: 7- and 
8-year-olds) based on the following rationale. The first age group is still developing their core language and ER. From the medium age group onwards, based on previous data (Widen \& Rusell, 2013), we would typically expect to observe the onset of ER and emotion language development. We expect this phenomenon to continue in the old group. At 8 years of age, we would expect hearing children to have a firm understanding of emotion labels and social-cognitive reasons for expressing different (including false) emotions.

The young group had $20 \mathrm{HI}$ children and 23 hearing children; the medium group $33 \mathrm{HI}$ children and 37 hearing children, and the old group $22 \mathrm{HI}$ children and 31 hearing children. As described in Table 1, Mann-Whitney's U test revealed that in all groups there were no differences between the children with and without hearing loss in cognitive ability scores or age.

Table 1. Mean age in months (and SD), and mean cognitive ability score (and SD) as a function of age group and hearing status

\begin{tabular}{lccllll}
\hline \multicolumn{7}{l}{ Cognitive ability } \\
& $\begin{array}{l}\text { HI } \\
\text { children }\end{array}$ & $\begin{array}{l}\text { Hearing } \\
\text { children }\end{array}$ & Comparison & $\begin{array}{l}\text { HI } \\
\text { children }\end{array}$ & $\begin{array}{l}\text { Hearing } \\
\text { children }\end{array}$ & Comparison \\
\hline Young group & 85.00 & 82.57 & $U=207.5$ & 48.8 & 47.96 & $U=211.5$ \\
$N=43$ & $(28.71)$ & $(30.47)$ & $p=.582$ & $(5.45)$ & $(5.49)$ & $p=.651$ \\
Medium group & 138.42 & 144.30 & $U=548.5$ & 70.15 & 70.73 & $U=579$ \\
$N=70$ & $(33.81)$ & $(25.57)$ & $p=.464$ & $(8.13)$ & $(7.56)$ & $p=.710$ \\
Old Group & 162.73 & 163.71 & $U=301$ & 92.5 & 93.52 & $U=320$ \\
$N=53$ & $(36.69)$ & $(20.76)$ & $p=.469$ & $(6.60)$ & $(7.4)$ & $p=.704$ \\
\hline
\end{tabular}

Data were analysed using IBM SPSS version 23.

\section{Results}

\section{Descriptive statistics}

First of all, we compared ER score between the groups with and without hearing loss (see Figure 1). Mann-Whitney's U test showed that while there were no significant differences between $\mathrm{HI}$ and hearing children in the young group ( $U=192.5, p=.351)$, differences in ER appeared in both the medium group $(U=368.5, p=.003)$ and the old group $(U=193, p=.002)$. Following Field (2009), we calculated the effect size of these comparisons (and of all Mann-Whitney comparisons in the study) with the formula $r=\mathrm{Z} / \sqrt{ } \mathrm{N}$. Effect sizes in the medium group were $r=0.36$, and $r=0.42$ in the old group. According to Cohen (1988, 
1992), when $r=0.1$, the effect size is small, when $r=0.3$ the effect is medium, and when $r=0.5$, the effect is large (see Field, 2009). Therefore, the abovementioned effects were medium.

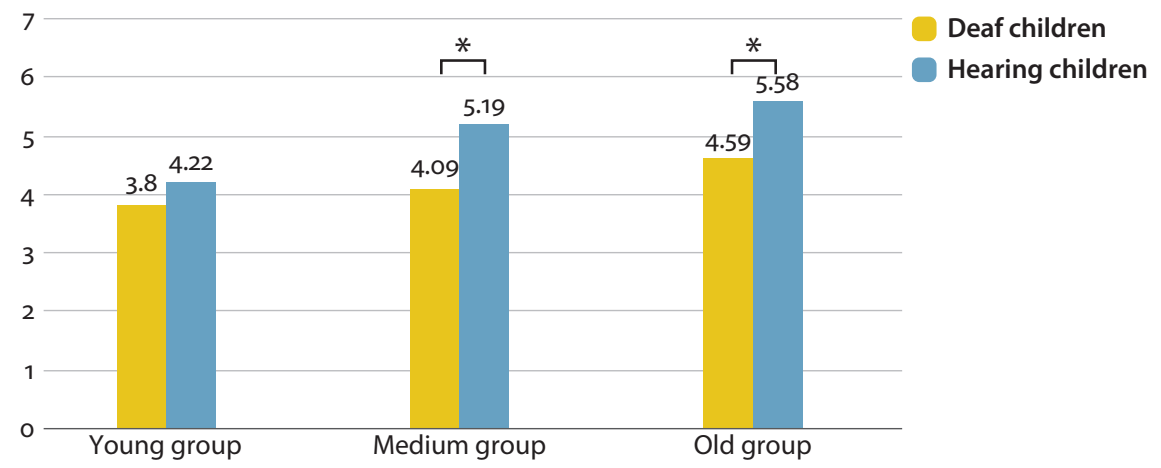

Figure 1. ER score (means) as a function of hearing status and age group

Note: Asterisks represent significant differences $(p<.005)$.

In relation to the linguistic variables, Mann-Whitney's $U$ test was used to compare the scores in vocabulary and LPP-2 (Total and subscales) between HI and hearing children in the three age groups (see Table 2). Results showed that: a) in the young group, HI children only showed lower scores than hearing children in the LPP-2 Form (medium effect size); b) in the medium group, HI children showed lower scores than hearing children in all the LPP-2 subscales, in the Total LPP-2 score, and in the vocabulary score (medium and large effect sizes); c) in the old group, HI children obtained lower scores in the LPP-2 subscales of Content, Reference and Cohesion (medium and large effect sizes), but not in the subscales of Form and Use. Significant differences were also found in this age group in the vocabulary score and in the total LPP-2 score.

Regarding audiological variables, HI children with cochlear implants obtained higher scores on ER $(N=36 ; M=4.25 ; S D=1.48)$ than children without cochlear implant $(N=39 ; M=4.08 ; S D=1.46)$, but these differences were not statistically significant $(U=655, p=.606)$. Regarding the level of hearing loss, we compared the score on ER between children with profound hearing loss $(N=37 ; M=4.16$; $S D=1.59$ ) and children with lower levels of hearing loss (mild, moderate and severe grouped together: $N=38 ; M=4.16 ; S D=1.35$ ). Mann-Whitney's $\mathrm{U}$ test showed no significant differences between the two groups. The combined effect of the variables cochlear implant and level of hearing loss could not be analyzed, as only 4 children with profound hearing loss did not have cochlear implant (while 33 had a CI), and only 3 children with lower levels of hearing loss had a cochlear implant (while 35 did not). 
Table 2. Means (and SD) of linguistic skills as a function of hearing status and age group

\begin{tabular}{|c|c|c|c|c|c|c|c|c|c|}
\hline & & Vocabulary & Form & Content & Reference & Cohesion & Use & LPP-2 Total & Summary \\
\hline \multirow{5}{*}{$\begin{array}{l}\text { Young } \\
\text { Group }\end{array}$} & $\mathrm{HI}$ & 99.45 & 72.08 & 67.34 & 65.79 & 45.21 & 61.23 & 63.44 & \multirow[t]{5}{*}{ Form: $\mathrm{HI}<\mathrm{H}$} \\
\hline & $N=20$ & $(20.67)$ & $(21.97)$ & (20.59) & $(20.13)$ & $(23.56)$ & $(22.45)$ & $(20.28)$ & \\
\hline & Hearing & 103.22 & 86.83 & 72.61 & 70.30 & 52.58 & 60.24 & 67.51 & \\
\hline & $N=23$ & $(16.12)$ & (11.88) & $(25.11)$ & $(17.58)$ & $(29.20)$ & $(19.81)$ & $(19.60)$ & \\
\hline & $\mathrm{U}$ & 193 & $\begin{array}{c}132^{*} \\
r=.37\end{array}$ & 180 & 206 & 202.5 & 226 & 203.5 & \\
\hline \multirow[t]{3}{*}{$\begin{array}{l}\text { Medium } \\
\text { group }\end{array}$} & $\begin{array}{l}\mathrm{HI} \\
N=32\end{array}$ & $\begin{array}{l}101.18 \\
(18.38)\end{array}$ & $\begin{array}{c}77.95 \\
(20.67)\end{array}$ & $\begin{array}{c}77.44 \\
(18.37)\end{array}$ & $\begin{array}{c}70.34 \\
(20.91)\end{array}$ & $\begin{array}{c}57.89 \\
(24.03)\end{array}$ & $\begin{array}{c}71.21 \\
(23.13)\end{array}$ & $\begin{array}{c}71.37 \\
(20.44)\end{array}$ & \multirow[t]{3}{*}{$\begin{array}{l}\text { In all variables: } \\
\mathrm{HI}<\mathrm{H}\end{array}$} \\
\hline & $\begin{array}{l}\text { Hearing } \\
N=37\end{array}$ & $\begin{array}{l}122.19 \\
(11.50)\end{array}$ & $\begin{array}{l}96.79 \\
(7.72)\end{array}$ & $\begin{array}{c}95.00 \\
(93.28)\end{array}$ & $\begin{array}{l}93.28 \\
(9.33)\end{array}$ & $\begin{array}{c}83.30 \\
(16.17)\end{array}$ & $\begin{array}{c}86.23 \\
(10.47)\end{array}$ & $\begin{array}{c}89.72 \\
(11.26)\end{array}$ & \\
\hline & $\mathrm{U}$ & $\begin{array}{c}207.5^{\star * *} \\
r=.56\end{array}$ & $\begin{array}{c}257.5^{* * *} \\
r=.53\end{array}$ & $\begin{array}{l}151^{\star * *} \\
r=.66\end{array}$ & $\begin{array}{c}188.5^{* * *} \\
r=.59\end{array}$ & $\begin{array}{l}238^{* * *} \\
r=.52\end{array}$ & $\begin{array}{l}363.5^{\star *} \\
r=.33\end{array}$ & $\begin{array}{l}227^{\star * *} \\
r=.46\end{array}$ & \\
\hline \multirow[t]{3}{*}{ Old group } & $\begin{array}{l}\mathrm{HI} \\
N=22\end{array}$ & $\begin{array}{l}112.36 \\
(16.76)\end{array}$ & $\begin{array}{c}82.59 \\
(18.29)\end{array}$ & $\begin{array}{c}86.74 \\
(12.24)\end{array}$ & $\begin{array}{c}79.73 \\
(14.38)\end{array}$ & $\begin{array}{c}67.57 \\
(26.59)\end{array}$ & $\begin{array}{c}83.21 \\
(16.88)\end{array}$ & $\begin{array}{c}79.65 \\
(15.86)\end{array}$ & \multirow{3}{*}{$\begin{array}{l}\text { Vocab. Conten } \\
\text { Ref. Coh. Total } \\
\text { LPP-2 HI }<\text { H }\end{array}$} \\
\hline & $\begin{array}{l}\text { Hearing } \\
N=31\end{array}$ & $\begin{array}{l}129.48 \\
(10.68)\end{array}$ & $\begin{array}{c}90.28 \\
(12.47)\end{array}$ & $\begin{array}{l}94.86 \\
(6.70)\end{array}$ & $\begin{array}{l}93.28 \\
(9.72)\end{array}$ & $\begin{array}{c}87.82 \\
(19.47)\end{array}$ & $\begin{array}{c}84.07 \\
(15.68)\end{array}$ & $\begin{array}{c}89.77 \\
(10.42)\end{array}$ & \\
\hline & $\mathrm{U}$ & $\begin{array}{c}133.5^{\star * *} \\
r=.52\end{array}$ & 258 & $\begin{array}{l}204.5^{*} \\
r=.35\end{array}$ & $\begin{array}{l}141^{* * * *} \\
r=.50\end{array}$ & $\begin{array}{l}167.5^{* *} \\
r=.44\end{array}$ & 327.5 & $\begin{array}{l}202.5^{\star} \\
r=.34\end{array}$ & \\
\hline
\end{tabular}

Note: "U" refers to Mann-Whitney's U scores. Asterisks represent: ${ }^{* *} p .<.001 ;{ }^{* *} p<.01 ;{ }^{*} p<.05 *$. HI stands for hearing-impaired children and $\mathrm{H}$ for hearing. Effect sizes of significant comparisons were calculated using " $r$ ". 


\section{Correlations between emotion recognition and language}

As it can be observed in Table 3, significant correlations (aged controlled) were found between ER and linguistic variables only in the HI group. Specifically, vocabulary and the LPP-2 subscales of Form, Reference and Use correlated with the ER score.

Table 3. Spearman partial correlations (age controlled) between emotion recognition, linguistic variables and cognitive ability

\begin{tabular}{llllllllll}
\hline & Vocab. & Form & Cont. & Ref. & Coh. & Use & $\begin{array}{l}\text { LPP-2 } \\
\text { Total }\end{array}$ & $\begin{array}{l}\text { Cog. } \\
\text { ability }\end{array}$ \\
\hline ER HI children $N=72$ & $.356^{* *}$ & $.254^{\star}$ & .227 & $.254^{\star}$ & .206 & $.296^{\star}$ & $.254^{\star}$ & .203 \\
ER hearing children $N=88$ & .053 & .089 & .133 & .036 & .057 & .124 & .105 & .145 \\
\hline
\end{tabular}

Note: Numbers correspond to " $r$ " values. Asterisks represent: ${ }^{\star *} p<.001 ;{ }^{* *} p<.01 ;{ }^{*} p<.05$.

Spearman partial correlations (aged controlled) between linguistic variables and ER were also carried out in each of the three age groups and separating the HI and hearing groups (see Table 4). In the group of HI children, significant correlations between linguistic variables and ER were found only in the medium age group. As far as the hearing group is concerned, we found two significant correlations with ER: a negative one with vocabulary in the young group, and a positive one with the Use subscale in the old group.

Table 4. Spearman partial correlations (age controlled) between emotion recognition, linguistic variables and cognitive ability as a function of age group

\begin{tabular}{lllllllllll}
\hline & & Vocab & Form & Content & Ref. & Coh. Use & $\begin{array}{c}\text { LPP-2 } \\
\text { Total }\end{array}$ & $\begin{array}{l}\text { Cognitive } \\
\text { ability }\end{array}$ \\
\hline Young & HI $N=17$ & .321 & -.069 & .057 & .009 & .047 & .247 & -.005 & .043 \\
Group & Hearing & $-.521^{*}$ & .175 & .214 & -.002 & .093 & .144 & .181 & .060 \\
& $N=20$ & & & & & & & & \\
Medium & HI $N=30$ & $.481^{* *}$ & $.447^{\star}$ & $.363^{\star}$ & $.393^{*}$ & .314 & $.391^{*}$ & $.398^{*}$ & .160 \\
group & Hearing & .073 & .166 & -.047 & -.093 & .119 & -.112 & -.027 & .034 \\
& $N=34$ & & & & & & & & \\
Old group & HI $N=19$ & .258 & .075 & -.074 & .135 & .121 & .209 & .168 & .429 \\
& Hearing & .119 & .004 & .174 & -.170 & .060 & $.414^{*}$ & .264 & .065 \\
& $N=28$ & & & & & & & & \\
\hline
\end{tabular}

Note: Numbers correspond to " $\mathrm{r}$ " values. Asterisks represent: ${ }^{* *} p<.001 ;{ }^{* *} p<.01{ }^{*} p<.05$. 


\section{Predictors of emotion recognition}

A linear regression analysis was conducted in order to find the predictors of ER using the forward method (see Table 5). Results showed that in hearing children the best predictor of ER was cognitive ability, while in HI children the best predictor of ER was vocabulary.

Table 5. Linear regression (forward method) with emotion recognition as a dependent variable, and linguistic variables (vocabulary, form, content, reference, cohesion, use and LPP-2 Total), cognitive ability, and age (in months) as predictors

\begin{tabular}{|c|c|c|c|c|c|c|c|c|}
\hline \multirow[t]{2}{*}{ Coefficients } & \multirow[b]{2}{*}{ Predictors } & \multirow[b]{2}{*}{$B$} & \multirow[b]{2}{*}{$\begin{array}{l}\text { Stand. } \\
\text { error }\end{array}$} & \multirow[b]{2}{*}{$\begin{array}{l}\text { Standarised } \\
\text { coefficients- } \\
\text { Beta }\end{array}$} & \multirow[b]{2}{*}{$t$} & \multirow[b]{2}{*}{ Sig. } & \multicolumn{2}{|c|}{ Summary of the model } \\
\hline & & & & & & & R squared & $\begin{array}{l}\text { Adjusted R } \\
\text { Squared }\end{array}$ \\
\hline \multirow{2}{*}{$\begin{array}{l}\text { HI children } \\
N=74\end{array}$} & Constant & .639 & .925 & & .691 & .492 & .170 & .158 \\
\hline & Vocabulary & .034 & .009 & .412 & 3.833 & .000 & & \\
\hline \multirow{2}{*}{$\begin{array}{l}\text { Hearing } \\
\text { children } \\
N=90\end{array}$} & Constant & 2.983 & .419 & & 7.119 & .000 & .238 & .230 \\
\hline & $\begin{array}{l}\text { Cognitive } \\
\text { ability }\end{array}$ & 0.16 & .003 & .488 & 5.247 & .000 & & \\
\hline
\end{tabular}

\section{Discussion}

There is great theoretical interest in the interaction between language and cognition (e.g. Gooch, Thompson, Nash, Snowling, \& Hulme 2016). While many aspects of language and cognition develop in parallel, it is not clear what the mutual influence is of one on the other. One way of looking at this is to explore contexts where one of these two factors is delayed significantly and to explore the effects of these delays on the other system. In previous work, language variables were found to predict ER score in HI but not hearing children (Sidera et al. 2017). The current study extends previous work by delineating how the diverse linguistic variables are related to ER at different developmental points. Our findings (see Table 3) support the view that different linguistic and communicative skills (especially the Form, Reference and Use of language, as well as vocabulary) are important for the development of ER (see Dyck et al. 2004). Correlations between language and ER were not found in hearing children when the whole group was considered, and when we divided it into 3 age groups, fewer correlations were found than in HI children. Nevertheless, this does not imply that language is less important for learning to recognise emotions in hearing children than in $\mathrm{HI}$ children. It could also be the case that the recognition of basic emotions is easily attained for children with a 
certain level of linguistic and communicative abilities. In the case of HI children, if these tools are not properly developed, the recognition of basic emotions might be affected too (Wang et al., 2016).

A criticism of our study could be that children's capacity to recognise emotions was measured using linguistic labels, thus it is possible that a non-verbal assessment of ER capacities might have led to different results. However, studies like Wang et al., (2016) or Wiefferink et al., (2013) have found differences between HI and hearing children even in non-verbal ER tasks, which also support the idea that a deficit in $\mathrm{HI}$ children in recognizing emotions is not just a linguistic problem related to labeling emotions in specific tasks, but a conceptual one. In this regard, Jones et al., (2017) reported that HI children are better with dynamic than with static faces. This suggests that increasing the saliency of the stimuli would lower their difficulties in ER. However, Jones et al. (2017) did not confirm that the HI children in their study had a linguistic delay. Furthermore if the moving face helped it was not for all emotions as HI children struggled with the dynamic expression of disgust. Many of the participants in Jones et al. (2017) were sign users, and it is not clear if results can be extended to non-signers. In any case, as these authors argue, children can learn about emotions in both formats (static and dynamic), so difficulties with ER in any format might be relevant. Future research should investigate differences in even more ecological situations, like videos of people expressing real emotions in specific contexts, rather than emotions from actors.

Our results that considered the different age groups separately showed that for the group of $\mathrm{HI}$ children different linguistic and communicative skills were important for ER, but only in the group of 5- and 6-year-olds. On the other hand, it is possible that correlations between language and ER were not observed in the young HI group because linguistic differences with the hearing group were minimal. In relation to the oldest group, despite linguistic differences between HI and hearing children, no correlations between language and ER were observed in this group. One possible interpretation is that most HI children at this age have already acquired the necessary linguistic tools for ER, so individual differences do not depend so much on language but on their emotion-related social experiences. In the case of hearing children, we found a negative correlation between vocabulary and ER in the young group and a correlation between Use of language and ER in the old group. Therefore, in the group of hearing children, the linguisticcommunicative skill from the LPP-2 most linked to ER was language Use. In this sense, the pragmatic aspect of language could be an important linguistic aspect used to learn to recognize emotions.

There are a couple of reasons why language use and ER are linked, which we propose here. The first is related to the developmental experience of hearing impairment which is generally very different to that of hearing children. In the 
first two years of life, typically developing children cultivate an understanding of other's intentions to communicate via joint attention and triadic interactions (Ninio \& Bruner, 1978; Tomasello \& Todd, 1983). The outcome of this early period of social-communicative routines is not only vocabulary development, but also emotion regulation. Consequently, a disruption to this early period of establishing meaningful interactions will have an impact on both language and emotion recognition (Carlson \& Wang, 2007; Botting et al., 2016). The second possibility (and not mutually exclusive) is that during the ER task itself children are mediating performance by self-directed speech. Previous work in hearing children with language delays has demonstrated that self-directed speech was less optimal and was a factor involved in reduced performance on similar tasks (Lidstone, Meins, \& Fernyhough, 2012).

More research concerning early experience of emotion talk in HI children is needed to explore these issues further. This could also help us to interpret the negative correlation found between vocabulary and ER in the youngest hearing group, while in the whole sample of HI children, vocabulary was found to be the best predictor of ER. In this respect, results might have been different if our vocabulary task had included vocabulary on emotions or mental states, as they have been found to correlate with ER tasks that involve labeling (Rieffe \& Wiefferink, 2017). According to Widen (2013), children's development of concepts referring to facial expressions of emotion (e.g. surprised, happy) does not only depend on vocabulary by connecting a label to a face but also on establishing relationships between different components of emotion understanding (e.g. understanding the causes and consequences of emotions). Finally, the small sample in the young HI group may also be a reason for such a correlation. Indeed, when a regression was carried out with the whole sample of hearing children, the best predictor of ER was not a linguistic variable, but cognitive ability.

As already mentioned, one limitation of our study is that, despite having a fairly large sample of $\mathrm{HI}$ children, this might not have been large enough to study the effects of different age groups. This was especially relevant when trying to study the effects of cochlear implant (CI) on ER skills. We did not find differences between $\mathrm{CI}$ and non CI children, but this result might have been obscured by the fact that the majority of children with CI had a profound hearing loss, while the majority of children without CI had better hearing. Another limitation of the present study may have been that the young HI children had a minimal linguistic delay. Future research could focus attention on this young group of children in order to detect which are the linguistic variables most intertwined with ER. Another interesting line of research refers to the origins of the difficulties in ER and emotion understanding in early communicative experiences. What are the conditions that promote this important social-cognitive development in the early 
parent-child interactions that happen daily in the first 12 months and how does hearing loss disrupt these early interactions leading to both language and socialcognitive delays?

In sum, we have observed that both formal language and different linguisticcommunicative skills (especially the use of language) play an important role in the development of ER. Due to the central role of this crucial aspect of social cognition in daily life, it is important that any delays are detected as soon as possible and remediation offered in order to improve the inclusion of HI children in wider society.

\section{Acknowledgements}

We thank the resource centres for children with hearing loss (CREDA) in Catalonia for their help, as well as speech therapists, teachers, schools and children who participated in the study.

This work was supported by the Spanish Ministerio de Economía y Competitividad and the Fondo Europeo de Desarrollo Regional (PSI2015-69419-R; MINECO-FEDER). Morgan's work was supported by the Economic and Social Research Council of Great Britain [grant number RES-620-28-0002], Deafness, Cognition and Language Research Centre (DCAL).

\section{References}

Arribas, D., \& Corral, S. 2011. BAS2, Escalas de aptitudes intelectuales. Madrid: TEA Ediciones. Astington, J., \& Jenkins, J. 1999. A longitudinal study of the relation between language and Theory of Mind development. Developmental Psychology 35, 1311-1320.

https://doi.org/10.1037/0012-1649.35.5.1311

Bebko, J. M., Calderon, R., \& Treder, R. 2003. The language proficiency profile-2: Assessment of the global communication skills of deaf children across languages and modalities of expression. Journal of Deaf Studies and Deaf Education 8, 438-451.

https://doi.org/10.1093/deafed/engo34

Bebko, J. M., \& McKinnon, E. E. 1993. The Language Proficiency Profile-2 (Unpublished assessment tool). York University, Toronto, Canada.

Botting, N., Jones, A., Marshall, C., Denmark, T., Atkinson, J., \& Morgan, G. 2016. Non-verbal executive function is mediated by language: A study of deaf and hearing children. Child Development 88(5), 1689-1700. https://doi.org/10.1111/cdev.12659

Carlson, S. M., \& Wang, T. S. 2007. Inhibitory control and emotion regulation in preschool children. Cognitive Development 22, 489-510.

https://doi.org/10.1016/j.cogdev.2007.08.002

Cohen, J. (1988). Statistical power analysis for the behavioural sciences (2nd ed.). New York: Academic Press

Cohen, J. (1992). A power primer. Psychological Bulletin, 112(1), 155-159. 
Dyck, M. J., Farrugia, C., Shochet, I. M. \& Holmes-Brown, M. 2004. Emotion recognition/ understanding ability in hearing or vision-impaired children: Do sounds, sights, or words make the difference? Journal of Child Psychology and Psychiatry 45, 789-800. https://doi.org/10.1111/j.1469-7610.2004.00272.x

Elliot, C. D., Smith, P., \& McCulloch, K. (1996) British ability scales (2nd ed.). Slough, UK: National Foundation of Educational Research.

Field, A. P. 2009. Discovering statistics using SPSS: And sex and drugs and rock ' $n$ ' roll (3rd ed.). London: Sage.

Gooch, D., Thompson, P., Nash, H. M., Snowling, M. J., \& Hulme, C. 2016. The development of executive function and language skills in the early school years. Journal of Child Psychology and Psychiatry 57, 180-187. https://doi.org/10.1111/jcpp.12458

Gray, C., Hosie, J., Russell, P., Banks, J., \& Ormel, E. 2001. Emotional development in deaf children: Understanding facial expressions, display rules, and theory of mind. In $\mathrm{M}$. Marschark, D. Clark, \& M. Karchmer (Eds.), Context, cognition, and deafness. Washington, DC: Gallaudet University Press.

Hopyan-Misakyan, T. M., Gordon, K. A., Dennis, M., \& Papsin, B. 2009. Recognition of affective speech prosody and facial affect in deaf children with unilateral right cochlear implants. Child Neuropsychology 15, 136-146. https://doi.org/10.1080/09297040802403682

Hosie, J., Gray, C., Russell, P., Scott, C., \& Hunter, N. 1998. The matching of facial expressions by deaf and hearing children and their production and comprehension of emotion labels. Motivation and Emotion 22, 293-313. https://doi.org/10.1023/A:1021352323157

Jones, A. C., Gutierrez, R., \& Ludlow, A. K. 2017. The role of motion and intensity in deaf children's recognition of real human facial expressions of emotion. Cognition and Emotion, 1-14. https://doi.org/10.1080/02699931.2017.1289894

Laugen, N. J., Jacobsen, K. H., Rieffe, C., \& Wichstrøm, L. 2017. Emotion understanding in preschool children with mild-to-severe hearing loss. The Journal of Deaf Studies and Deaf Education 22(2), 155-163. https://doi.org/10.1093/deafed/enwo69

Lidstone, J. S. M., Meins, E., \& Fernyhough, C. 2012. Verbal mediation of cognition in children with specific language impairment Development and Psychopathology 24, 651-660. https://doi.org/10.1017/So954579412000223

Mancini, P., Giallini, I., Prosperini, L., D’alessandro, H. D., Guerzoni, L., Murri, A., ... Nicastri, M. 2016. Level of emotion comprehension in children with mid to long-term cochlear implant use: How basic and more complex emotion recognition relates to language and age at implantation. International Journal of Pediatric Otorhinolaryngology 87, 219-232. https://doi.org/10.1016/j.ijporl.2016.06.033

Meristo, M., Strid, K., \& Hjelmquist, E. 2016. Early conversational environment enables spontaneous belief attribution in deaf children. Cognition 157, 139-145.

https://doi.org/10.1016/j.cognition.2016.08.023

Morgan, G., Meristo, M., Mann, W., Hjelmquist, E., Surian, L., \& Siegal, M. 2014. Mental state language and quality of conversational experience in deaf and hearing children. Cognitive Development 29, 41-49. https://doi.org/10.1016/j.cogdev.2013.10.002

Most, T., \& Aviner, C. 2009. Auditory, visual, and auditory-visual perception of emotions by individuals with cochlear implants, hearing aids, and normal hearing. Journal of Deaf Studies and Deaf Education 14, 449-464. https://doi.org/10.1093/deafed/enpoo7 
Most, T., \& Michaelis, H. 2012. Auditory, visual, and auditory-visual perception of emotions by young children with hearing loss versus children with normal hearing. Journal of Speech, Language, and Hearing Research 55, 1148-1162. https://doi.org/10.1044/1092-4388(2011/11-0060)

Ninio, A., \& Bruner, J. 1978. The achievement and antecedents of labeling. Journal of Child Language 5(1), 1-15. https://doi.org/10.1017/S0305000900001896

Peterson, C. C., Wellman, H. M., \& Slaughter, V. 2012. The mind behind the message: Advancing theory of mind scales for typically developing children, and those with deafness, autism, or Asperger syndrome. Child Development 83(2), 469-485.

Rieffe, C., \& Wiefferink, C. H. 2017. Happy faces, sad faces: Emotion understanding in toddlers and preschoolers with language impairments. Research in Developmental Disabilities 62, 40-49.

Sidera, F., Amadó, A. \& Martínez, L. 2017. Influences on facial emotion recognition in deaf children. The Journal of Deaf Studies and Deaf Education 22(2), 164-177. https://doi.org/10.1093/deafed/enwo72

Tomasello, M., \& Todd, J. 1983. Joint attention and lexical acquisition style. First Language 4, 197-212. https://doi.org/10.1177/014272378300401202

Wang, Y., Su, Y., Fang, P., \& Zhou, Q. 2011. Facial expression recognition: Can preschoolers with cochlear implants and hearing aids catch it? Research in Developmental Disabilities 32, 2583-2588. https://doi.org/10.1016/j.ridd.2011.06.019

Wang, Y., Su, Y., \& Yan, S. 2016. Facial expression recognition in children with cochlear implants and hearing aids. Frontiers in Psychology 7, 1989. https://doi.org/10.3389/fpsyg.2016.01989

Widen, S. C. 2013. Children's interpretation of facial expressions: The long path from valencebased to specific discrete categories. Emotion Review 5(1), 72-77. https://doi.org/10.1177/1754073912451492

Widen, S. C., \& Russell, J. A. 2013. Children's recognition of disgust in others. Psychological Bulletin 139, 271-299. https://doi.org/10.1037/aoo31640

Wiefferink, C. H., Rieffe, C., Ketelaar, L., De Raeve, L., \& Frijns, J. H. 2013. Emotion understanding in deaf children with a cochlear implant. Journal of Deaf Studies and Deaf Education 18, 175-186. https://doi.org/10.1093/deafed/enso42

Ziv, M., Most, T., \& Cohen, S. 2013. Understanding of emotions and false beliefs among hearing children versus deaf children. Journal of Deaf Studies and Deaf Education 18, 161-174. https://doi.org/10.1093/deafed/enso73 